\title{
Magnetic turbulent spectra in the magnetosheath: new insights
}

\author{
F. Sahraoui ${ }^{1}$, G. Belmont ${ }^{1}$, J. L. Pinçon ${ }^{2}$, L. Rezeau ${ }^{1,3}$, A. Balogh ${ }^{4}$, P. Robert ${ }^{1}$, and N. Cornilleau-Wehrlin ${ }^{1}$ \\ ${ }^{1}$ Centre d'Etude des Environnements Terrestre et Planétaires, 10/12 avenue de l'Europe, 78140, Vélizy, France \\ ${ }^{2}$ Laboratoire de Physique et de Chimie de l'Environnement, 3A avenue de la Recherche Scientifique, Orléans, France \\ ${ }^{3}$ Also at Université Pierre et Marie Curie, Paris, France \\ ${ }^{4}$ Space and Atmospheric Group, The Blackett laboratory, Imperial College, Prince Consort road, London, UK
}

Received: 24 February 2004 - Revised: 6 April 2004 - Accepted: 20 April 2004 - Published: 14 June 2004

\begin{abstract}
The spectrum of the magnetic fluctuations measured by the Cluster satellites in the inner magnetosheath is investigated using the k-filtering technique. On a case study, it is shown first that the wave vectors calculated from the Flux Gate Magnetometer (FGM) data fit well with those determined from the Spatio-Temporal Analysis of Field Fluctuations (STAFF) data for their common range of frequency, which allows one to confirm that the high pass filter applied to STAFF data does not alter the spatial characteristics of its spectra. Both analyses confirm the dominance of the mirror mode for frequencies up to $1.4 \mathrm{~Hz}$. Furthermore, by comparing the experimental charateristics of the identified mirror mode to the prediction of the linear theory, it is shown that the predicted maximum growth rate is observed in the frequency range $0-0.15 \mathrm{~Hz}$, i.e. the FGM range. All the rest of the mirror mode, identified for higher frequencies is more likely to be a non linear extension of the most instable one. This cascade on the spatial scales is, in turn, observed in the satellite frame as a temporal spread due to Doppler shift. Further implications on the real nature of the observed spectrum are discussed.
\end{abstract}

Key words. Magnetospheric physics (magnetosheath, plasma waves and enstabilities). Space plasma phusics (turbulence)

\section{Introduction}

The magnetic turbulence in the terrestrial magnetosheath plays a key role in the dynamical coupling between the solar wind and the magnetosphere. It has been investigated for many years both experimentally (Hubert et al., 1989; Gleaves and Southwood , 1991; Song et al., 1994; Denton et al., 1995; Constantinescu et al., 2003) and theoretically (Omidi and Winske, 1995; Génot et al., 2001; Hellinger et al., 2003). Now, thanks to the availability of the multipoint measure- ments of Cluster, we are able to investigate the 3-D properties of the observed turbulence in a more realistic way, and to go further in understanding the deep embedded physics. This, of course, is possible, providing one uses well adapted tools that take full advantage of the 3-D Cluster data. The $\mathrm{k}$-filtering technique, which was introduced in the context of space physics by Pinçon and Lefeuvre (1991), was applied by Sahraoui et al. (2003) to study the magnetic fluctuations measured by the Cluster-STAFF experiment. In this first application of the k-filtering technique to STAFF data, they have discussed the new opportunities that it offers to study the space plasma turbulence. They have, for the first time, directly demonstrated the complex behaviour of the ULF magnetic field fluctuations: each observed frequency in the satellite frame corresponds to a superposition of several plasma modes, with different wave vectors, and therefore, different frequencies in the plasma frame. They have particularly shown that, in the frequency range $0.35-1.2 \mathrm{~Hz}$, most of the energy is due to the mirror instability, but this does not prevent the existence of other plasma modes (Alfvén, slow, cyclotron, ...) with weaker energies. However, they also pointed out a discrepancy between the identified mirror mode and the prediction of its linear theory: the $\mathbf{k}$ modulus of the most intense mirror mode, observed in the interval $0.35-1.2 \mathrm{~Hz}$, is larger than the theoretical maximum growth rate. The fundamental aim of the present paper is to explain the mentioned discrepancy between theory and observations. In Sect. 2, we present the new data from the FGM experiment that are used in the present work; in Sect. 3 we briefly recall the principle of the k-filtering method and present its application to compare wave mode identifications from FGM and STAFF experiments. Section 4 is dedicated to the comparison of the obtained charateristics of the mirror mode to the prediction of the linear theory. Some new implications on the observed magnetic spectrum are then discussed. 

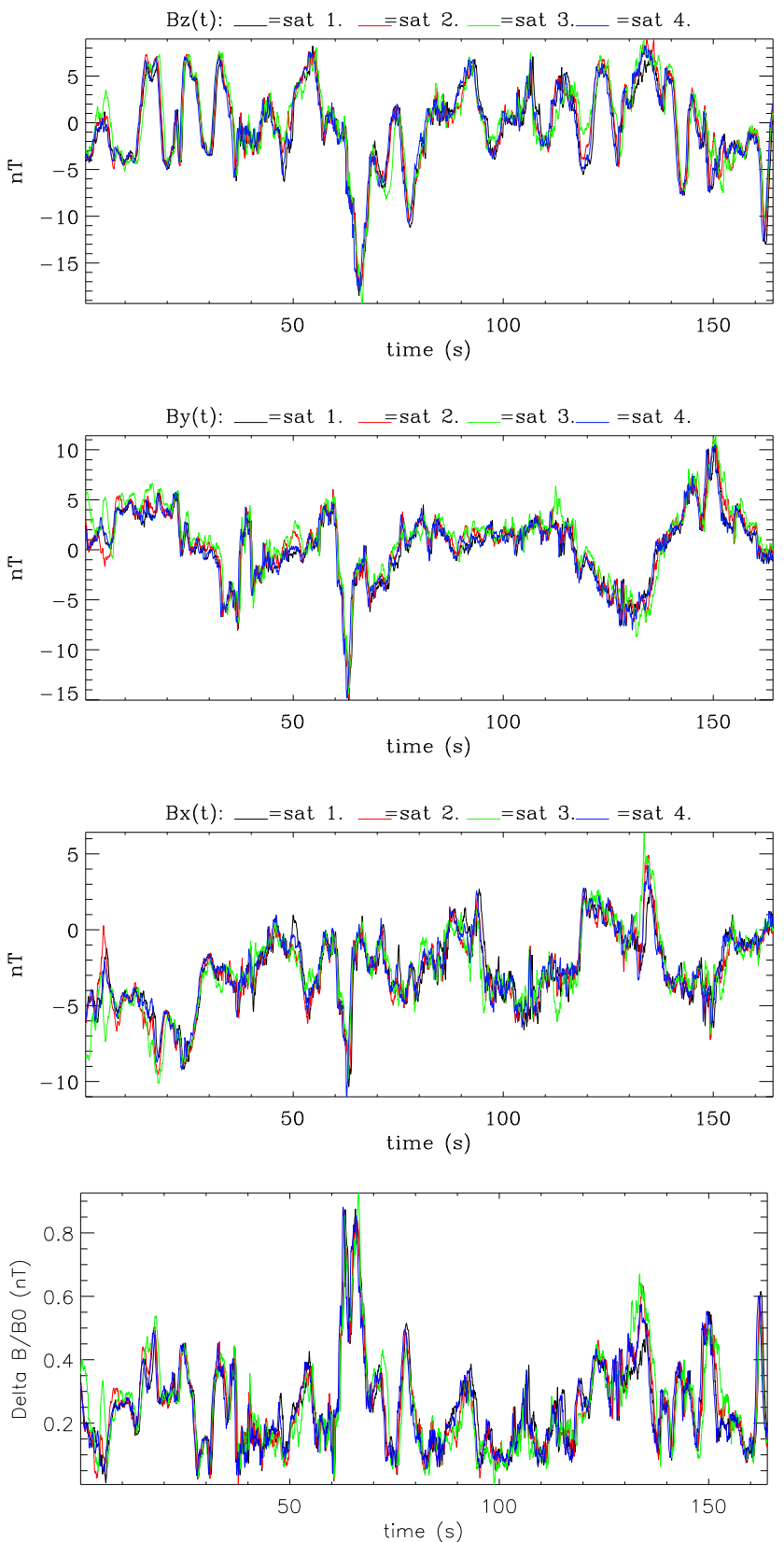

Fig. 1. The magnetic field components measured by the FGM experiment in the MFA frame. The continuous components are removed from the data and used to define the MFA frame. The three first panels (from top to bottom) represent respectively the parallel and the perpendicular components of the magnetic fluctuations. The last one shows the modulus of the magnetic fluctuations normalized to the background magnetic field. The color code is relative to the four satellites.

\section{Observations}

The data used in the present study have been gathered by the Cluster spacecraft on 18 February 2002 in the inner magnetosheath around 05:34 (LT). The crossing of the magnetopause by the spacecraft was about $35 \mathrm{~min}$ before. The
FGM data are sampled at $23 \mathrm{~Hz}$, and allow one to access the continuous components of the magnetic field (Balogh et al., 1997). These continuous components are removed from the signal and used to define the Magnetic Field Aligned (MFA) frame, in which this study is performed. The $\mathrm{Z}$ axis of the MFA frame is aligned along $\mathbf{B}_{0}$. For this event, the STAFF Search Coil data are sampled at $25 \mathrm{~Hz}$. Once the data are calibrated and despun, the signal is recovered safely on the full three components for frequencies above $0.35 \mathrm{~Hz}$ (CornilleauWehrlin et al., 1997). The lower frequencies are also accessible but this requires much more caution because of the satellite spin at $0.25 \mathrm{~Hz}$. Therefore, to avoid any problems relative to this last point, we use the FGM data to prolong the study to frequencies below $0.35 \mathrm{~Hz}$. In the present work, we investigate the magnetic turbulence in the frequency range $0.2 \mathrm{~Hz}$ : FGM data are used from 0 to $0.35 \mathrm{~Hz}$, whereas STAFF data are used from $0.35-2 \mathrm{~Hz}$. This will allow one to complete the first study done by Sahraoui et al. (2003), which used STAFF data only and analysed the frequency range $0.35-1.2 \mathrm{~Hz}$. Now, we will particularly focus on the frequency range $0,0.5 \mathrm{~Hz}$, and two main goals are checked: first we check whether the high pass filter (with $f_{\text {cut }- \text { off }}=0.35 \mathrm{~Hz}$ ) that had been applied to STAFF data did not alter the physical results provided by the $\mathrm{k}$-filtering method. This is done by comparing the nature of the identified waves in the frequency range $0.35-0.5 \mathrm{~Hz}$ covered by both experiments. Then, we will study from FGM data the nature of the new waves that could appear for frequencies lower than $0.35 \mathrm{~Hz}$. This last purpose will allow, as we can see below, one to answer some questions that have been raised in the first work of Sahraoui et al. (2003).

In Fig. 1 the X, Y, and Z magnetic waveforms from FGM experiment in the MFA frame over about $164 \mathrm{~s}$ are shown. As one can see, the parallel component $B_{z}$ shows low amplitude oscillations of about a 10-s period, more clearly observable during the first $60 \mathrm{~s}$ and between 90 to $150 \mathrm{~s}$.

These dominant oscillations can also be seen on the FFT spectrum of the parallel component shown in Fig. 2. In fact, up to $0.2 \mathrm{~Hz}$, the parallel component dominates the perpendicular one with a significant enhancement around the frequency $0.1 \mathrm{~Hz}$. Between 0.2 to $0.6 \mathrm{~Hz}$, the parallel component is slightly larger than the perpendicular one and exibits more oscillations. From 0.6 up to $2 \mathrm{~Hz}$ the two components have comparable levels and both exhibit small peaks. The dominance of the parallel component in the LF part of the spectrum can be taken as a signature of compressible waves. These issues will be clarified in the next section.

\section{Wave identification using the k-filtering technique}

From the measure of a given wave field simultaneously in several points in space, the $\mathrm{k}$-filtering technique allows one to estimate the corresponding energy distribution in the 4D Fourier space $(\omega, \mathbf{k})$, namely the function $P(\omega, \mathbf{k})$. The k-filtering method adopts a plane wave decomposition and requires the time stationarity and space homogeneity of the 


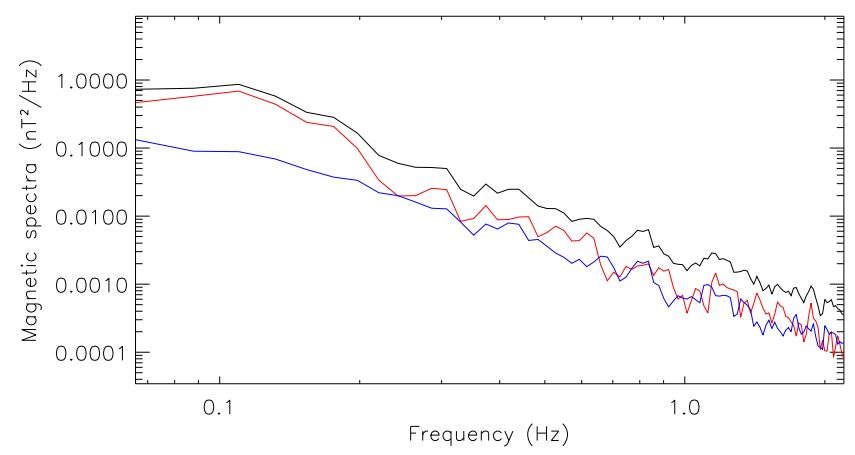

Fig. 2. The FFT spectra calculated from FGM data for the frequency range $[0.07,2] \mathrm{Hz}$. The parallel component (red line) is compared to the perpendicular one (blue line), and to the whole spectrum (black line).

time series. In real data, these two hypotheses are never strictly fulfilled. However, in practice, we are content with the concept of weak space (time) homogeneity (stationarity): the signal should be homogeneous (stationary) on scales that are larger than the largest spatial (temporal) scale determined by the k-filtering method. Obviously, the determination of the wave vectors $\mathbf{k}$ from spatially undersampled data (even the Cluster ones) is not trivial. The k-filtering technique uses a sophisticated method to overcome this difficulty: it introduces nonlinear filters for each couple $(\omega, \mathbf{k})$, requiring that all the energy contained in the signal is absorbed except that related to $(\omega, \mathbf{k})$ (Pinçon and Motschmann, 1998). The validity domain of the technique in the wave vector space is determined from the separations between the Cluster satellites: all the existing wavelengths have to be larger than the spacecraft separations, which are of the order of $100 \mathrm{~km}$ in the present case. Once the magnetic energy distribution $P(\omega, \mathbf{k})$ is calculated, it can be used to identify the existing propagating modes (Sahraoui et al., 2003): for each given frequency $\omega_{0}$, and using an isocontour representation, the distribution of energy in $\mathbf{k}$-space is displayed as cuts in the $\left(k_{x}, k_{y}\right)$ plane along the $k_{z}$ axis. For each $k_{z}$ corresponding to an identified peak, the theoretical dispersion relations of the plasma LF linear modes (MHD and mirror modes) are then superposed after being Doppler shifted using the flow velocities from the CIS experiment (Rème et al., 1997). The mirror mode is assumed to have approximately a zero frequency in the plasma frame, which means that it is observed in the satellite frame with the dispersion $\omega=\mathbf{k . v}$. The dispersion relations are computed using the WHAMP program (Rönnmark, 1982), where the control parameters are those measured by the different Cluster experiments: $\mathbf{B}_{0}$ from FGM, ion temperatures from CIS, and plasma density from WHISPER (Décreau et al, 1997). For more details on the previous results, the reader is referred to Sahraoui et al. (2003). Application of the k-filtering technique to the frequency $\mathrm{f}=0.44 \mathrm{~Hz}$, accessible on FGM and STAFF data, allows for the identification of an isolated peak shown in Fig. 3. The energy distributions determined from the two experiments look al-
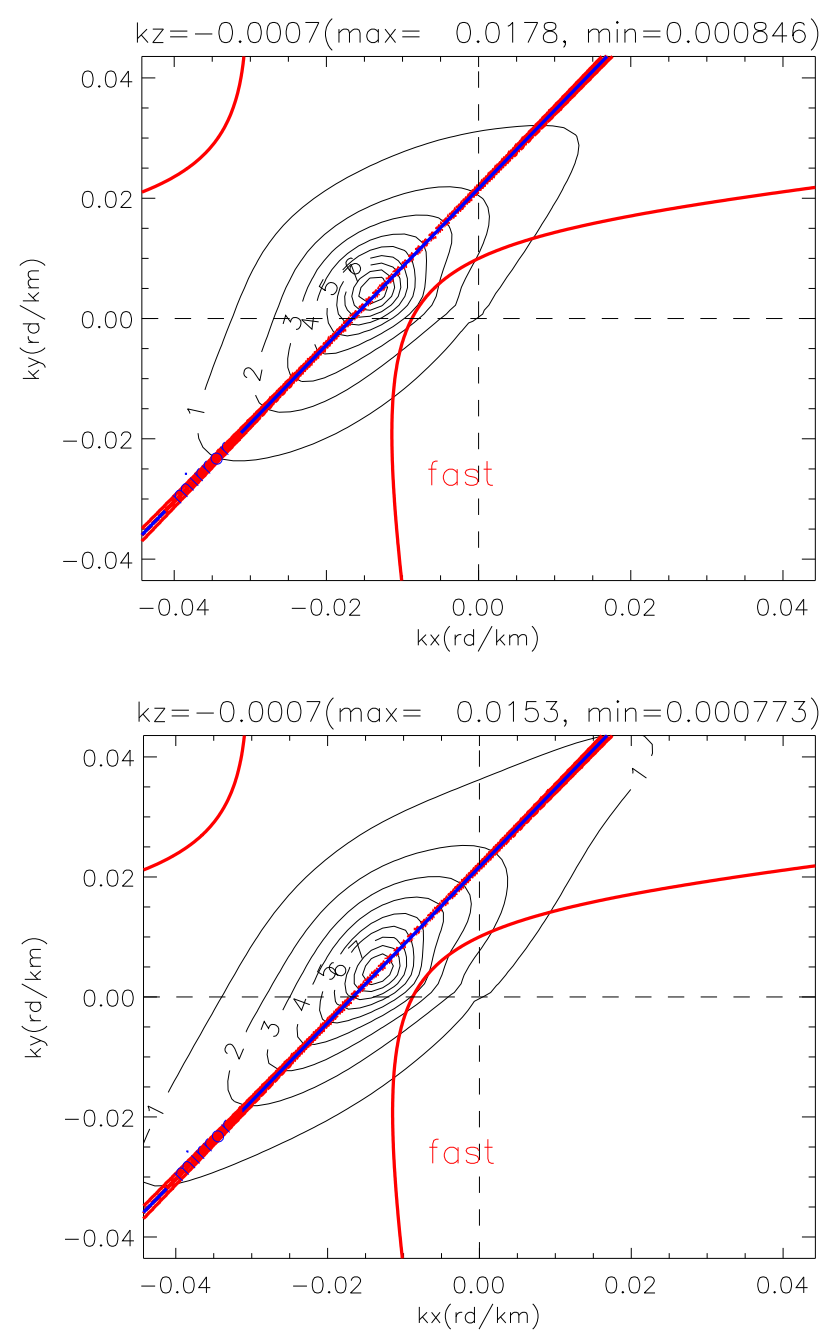

Fig. 3. Comparison of the energy distribution in $\mathbf{k}$ space of the most intense identified peak for the frequency $\mathrm{f}=0.44 \mathrm{~Hz}$ from $\mathrm{FGM}$ (top panel) and STAFF (bottom one). The black thin lines are the isocontours of energy in the $\left(k_{x}, k_{y}\right)$ plane, whereas the colored lines are the theoretical dispersion relations of the LF modes. The blue line is the Doppler shift $\omega=\mathbf{k} . \mathbf{v}$ and corresponds to the mirror dispersion relation in the satellite frame. Alfvén and slow modes (red lines) are very close to the mirror dispersion curve in this case of a quasi-perpendicular direction of propagation.

most identical: the two peaks are, respectively, centred on the wave vectors $\mathbf{k}_{F G M}=(-142.5,51.7,-7.1) \times 10^{-4} \mathrm{rd} / \mathrm{km}$ and $\mathbf{k}_{\mathrm{STAFF}}=(-127.5,51.7,-7.1) \times 10^{-4} \mathrm{rd} / \mathrm{km}$. The two wave vectors are separated by an angle less than $3^{\circ}$. This similarity is also proven for other frequencies (not shown here). This very satisfactory result demonstrates that dropping the low frequency part of the spectrum from STAFF data does not alter the physical results provided by the kfiltering technique at higher frequencies. By combining data from the two experiments, this last point allows one to take, in particular, full advantage of each experiment in the corresponding frequency range where its functioning is the best (Alexandrova et al., 2004). 


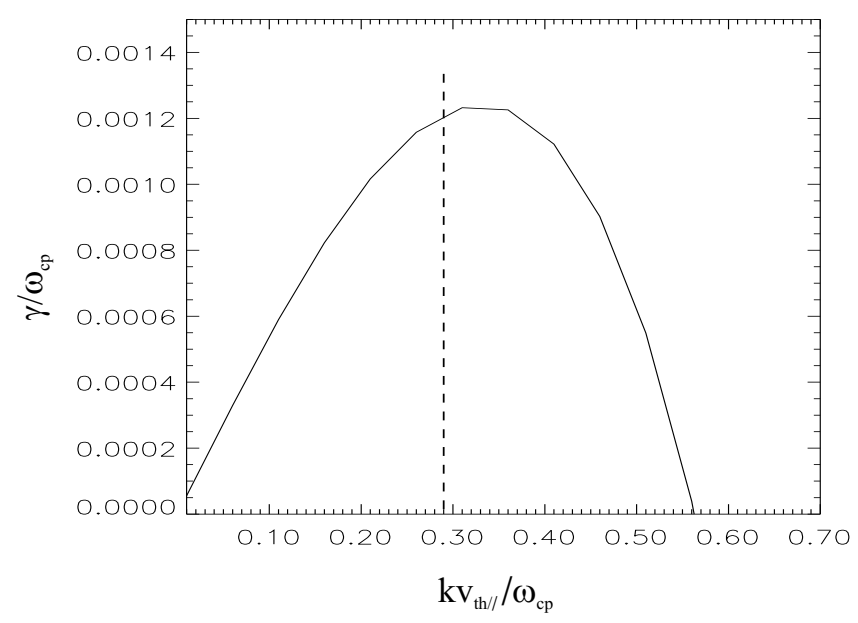

Fig. 4. The linear growth rate calculated from WHAMP using the measured parameters for the angle $\left(\mathbf{k}, \mathbf{B}_{0}\right)=80^{\circ} . \mathrm{v}_{\mathrm{th} / /}=154 \mathrm{~km} / \mathrm{s}$ is the parallel thermal velocity of the protons, $\omega_{\mathrm{cp}}=2.07 \mathrm{rd} / \mathrm{s}$ is their cyclotron pulsation. The vertical dashed line shows the location of the experimental wave vector of the most intense identified mirror mode.

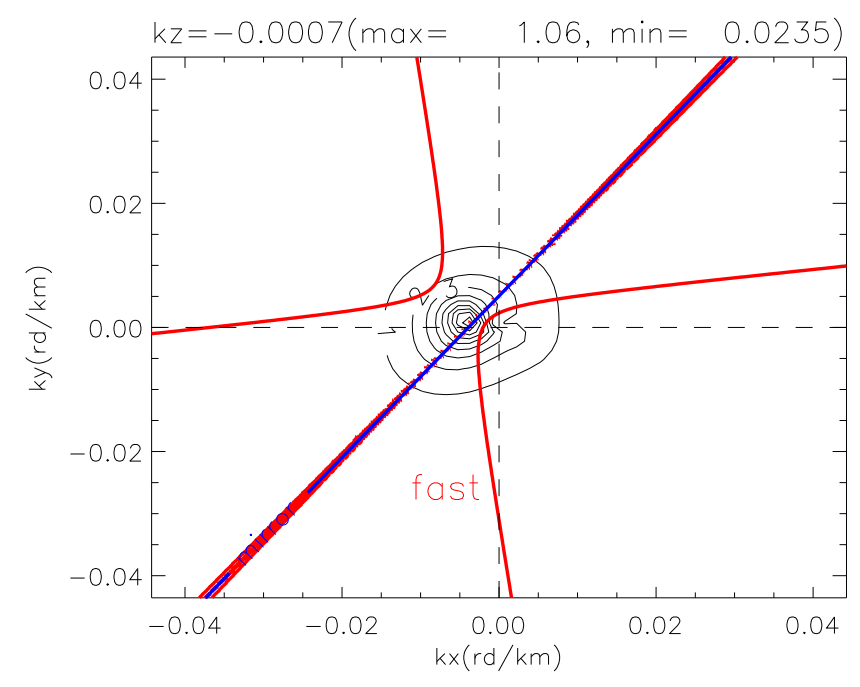

Fig. 5. The most intense peak identifiable on the whole spectrum $0-10 \mathrm{~Hz}$. It is observed at $\mathrm{f}=0.11 \mathrm{~Hz}$ centred on the mirror mode dispersion curve $\omega=\mathbf{k . v}$ (blue curve). Its wave vector $\mathbf{k}$ has an angle $-80^{\circ}$ with $\mathbf{B}_{0}$ and a modulus $\mathrm{k}=0.00389 \mathrm{rd} / \mathrm{km}$. Alfvén and slow modes (straight red curve) are degenerated and very close to $\omega=\mathbf{k . v}$.

\section{Discussion}

Now let us look at the physical properties of the whole spectrum: $0-0.35 \mathrm{~Hz}$ from FGM and $0.35-2 \mathrm{~Hz}$ from STAFF. As we can see in Fig. 3, which shows the k-energy distribution for the observed frequency $\mathrm{f}=0.44 \mathrm{~Hz}$, the identified peak is localized on the line $\omega=\mathbf{k . v}$ which corresponds to the dispersion relation of the mirror mode in the satellite frame $\left(\omega \sim 0\right.$ in the plasma frame). Its wave vector makes a $-80^{\circ}$ angle with respect to the local magnetic field.
From a theoretical point of view, it is well established that the mirror instability grows in a hot plasma because of the protons' temperature anisotropy and when the criterion $A_{p}-1 \geq 1 / \beta_{\perp}$ is fulfilled, where $A_{p}=T_{p_{\perp}} / T_{p_{/ /}}$is the proton anisotropy parameter (Hasegawa., 1975; Gedalin et al., 2002; Hellinger et al., 2003). Using WHAMP and the physical parameters measured in the context of this study, we calculate the linear growth rate of the mirror instability. For this purpose, we assumed a maxwellian distribution functions for the protons. The electrons are assumed isotropic with a temperature $T_{e}=10 \mathrm{eV}$, whereas the measured protons anisotropy is $A_{p} \sim 1.28$.

In Fig. 4 the linear growth rate of the mirror instability is shown as a function of the wave vector for the angle $\theta=\left(\mathbf{k}, \mathbf{B}_{0}\right)=80^{\circ}$.

The theoretical maximum growth rate is obtained for the value $k_{\gamma_{\max }}=0.004 \mathrm{rd} / \mathrm{km}$. Although the measured parameters are very close to the marginal instability threshold $\left(A_{p} \sim 1.28, \beta_{\perp} \sim 4\right)$, the theoretical value is three times less than the observed modulus of the mirror mode wave vector $\mathrm{k}=0.0124 \mathrm{rd} / \mathrm{km}$ associated with the satellite frame frequency $(\mathrm{f}=0.37 \mathrm{~Hz})$ studied by Sahraoui et al. (2003). This discrepancy between the observations and the basic linear theory of the mirror mode was pointed out in that work, and has remained unanswered since then.

Now, to solve this problem, we notice first that the mirror mode is observed in the quasi-perpendicular direction, its dispersion relation can be written as $\omega_{\mathrm{obs}}=\mathbf{k . v} \sim k_{\perp} v_{\perp}$. Accordingly, one may expect to observe increasing $k_{\perp}$ with increasing frequencies in the satellite reference frame. This point was indeed verified in Sahraoui et al. (2003), since the identified peaks have larger $k$ for higher frequencies in the frequency range $0.35-1.2 \mathrm{~Hz}$. These observations, therefore, support that the mirror instability would actually develop for frequencies lower than $0.35 \mathrm{~Hz}$, and would be observable for higher frequencies only by Doppler shift.

To check the validity of this interpretation, we look at the results of the k-filtering technique obtained in the frequency range $0,0.35 \mathrm{~Hz}$ from FGM data. The most intense peak is identified at the frequency $\mathrm{f}=0.11 \mathrm{~Hz}$ in the satellite reference frame, which corresponds to the 10 -s period oscillating waves previously seen on the parallel component of the fluctuations (Fig. 1) and explains the energy enhancement on the parallel spectrum at $f \sim 0.1 \mathrm{~Hz}$ (Fig. 2). This most intense peak, shown in Fig. 5, lies on the dispersion curve of the mirror mode $\omega=\mathbf{k . v}$. The corresponding wave vector $\mathbf{k}$ makes $\mathrm{a}-80^{\circ}$ angle with $\mathbf{B}_{0}$ and its modulus is $\mathrm{k}=0.00389 \mathrm{rd} / \mathrm{km}$. These experimental characteristics compare very favorably to the prediction of the linear kinetic theory of the mirror instability. As it is shown in Fig. 4, the measured wave vector of the mirror mode at the frequency $\mathrm{f}=0.11 \mathrm{~Hz}$ is very close to the one associated with the theoretical maximum growth rate of the mirror instability. Although this mirror mode is generated at such a spatial scale for the zero frequency in the plasma frame, it is still observed in the satellite frame up to $1.4 \mathrm{~Hz}$ with decreasing wavelengths and energy (Figs. 5 and 3). This nonlinear extension of the mirror spectrum turns 
out to be observed in the satellite frame as a temporal effect by Doppler shift. The mirror mode spectrum could also extend to frequencies higher than $1.4 \mathrm{~Hz}$, but the corresponding wave vectors are not accessible to measurement because of the limitation imposed by the Cluster separations.

Besides the previous mirror mode, other modes, with lower intensities, are also identifiable by the k-filtering method (Sahraoui et al., 2003). The existence of other modes, having a shear magnetic component, is indeed expected from Fig. 2, where the perpendicular component of the spectrum exhibits small fluctuations and reaches the level of the parallel one around $\mathrm{f}=0.7 \mathrm{~Hz}$. This was indeed confirmed by identifying Alfvén/cyclotron modes above $0.6 \mathrm{~Hz}$, their intensities compete with that of the previous mirror mode for increasing frequencies. The identification of these cyclotron modes is based only upon the calculation of their frequencies in the plasma frame, which are multiples of the proton gyrofrequency. The cylotron modes also exist for frequencies up to $\mathrm{f}=1.8 \mathrm{~Hz} \sim 6 f_{\mathrm{cp}}\left(f_{\mathrm{cp}} \sim 0.3 \mathrm{~Hz}\right.$ is the proton gyrofrequency), but their study requires much caution because of the weak level of energy at such high frequencies. A more refined study of these cyclotron modes, including their possible interaction with the mirror one, is postponed to a future work.

\section{Conclusions}

A case study of a magnetic spectrum in the magnetosheath using the k-filtering method is presented. It is shown that magnetic data from STAFF and FGM experiments are complementary and provide very similar results concerning the modes that propagate the magnetic energy. Thanks to this continuity of the results over the frequency range covered by each experiment, a new image of a high beta magnetic spectrum is obtained. In the same line with the results published by Sahraoui et al. (2003), it is shown that the LF part of the observed spectrum is dominated by the mirror mode having quasi-perpendicular wave vectors with respect to the static magnetic field. It is shown that the magnetic energy seems to be injected at a spatial scale that is in very good agreement with the predicted maximum growth rate of the mirror instability. Even if this energy injection, via the linear mirror instability, is observed at the lowest frequency part of the spectrum, the mirror mode is nevertheless still observed, at higher frequencies in the satellite frame, with decreasing wavelengths. This spatial extension, from the longest wavelength corresponding to the maximum growth rate to the shorter ones, is certainly due to nonlinear effects. It can indeed be viewed as a classical turbulent cascade, from large to small scales, or as a nonlinear saturation of the mirror instability, evoking more coherent effects.

The logical continuations of the present work are, first, to carry out such a continuous $\mathbf{k}$-spectrum by integrating over all the frequencies for which the mirror mode is observed. The second step will be to try to answer the question of the origin of the resulting $\mathbf{k}$-spectrum (turbulent cascade or sat- uration). If the cascade scenario is confirmed, a theoretical explanation of this new "hydrodynamic mirror turbulence" will have to be built to interpret the observed spatial cascade. This work is in progress, and will be the subject of a future publication.

Acknowledgements. The first author of this work was funded by a CNES followship.

The authors would to thank J. M Bosqued and P. Canu, respectively from CIS and WHISPER teams, for providing the data used in this work to compute the physical parameters of the plasma.

Topical Editor T. Pulkkinen thanks D. Winske for his help in evaluating this paper.

\section{References}

Alexandrova, O., Mangeney, A., Maksimovic, M., Lacombe, C., Cornilleau-Wehrlin, N., Lucek, E. A., Décréau, P. M. E, Bosqued, J. -M, Travnicek, P., and. Fazakerley, A. N: Cluster observations of finite-amplitude Alfvén waves and small-scale filaments downstream of a quasi-perpendicular shock, J. Geophys. Res., vol. 109, A05207, doi10.1029/2003JA 010056, 2004.

Balogh, A., Dunlop, M. W., and Cowley, S. W. H. et al.: The Cluster Magnetic field Investigation, Sp. Sci. Rev., 79, 65-91, 1997.

Constantinescu O. D., Glassmeier, K. H., Treumann, R., and Fornaçon, K. H.: Magnetic mirror structures observed by Cluster in the magnetosheath, Geophys. Res. Lett, 30, doi: 10.1029/2003GL017313, 2003.

Cornilleau-Wehrlin, N., Chauveau, S., and Louis, S., et al.: The Cluster spatio-temporal analysis of field fluctuations (STAFF) experiment, Sp. Sci. Rev., 79, 107-136, 1997.

Décréau P. M. E., Fergeau, P., and Krasnoselsk'kikh, V., et al.: WHISPER, A resonance sounder and wave analyser: performances and perspectives for the Cluster mission, Sp. Sci. Rev., 79, 157-193, 1997.

Denton, R. E., Gary, S. P., Li, X., Anderson, B. J., Labelle, J. W., and Lessard, M.: Low-frequency fluctuations in the magnetosheath near the magnetopause, J. Geophys. Res., 100, 56655679, 1995.

Gedalin, M., Balikhin, M., Strangeway, R. J, and Russel, C. T.: Long-wavelength mirror modes in multispecies plasmas with arbitrary distributions, J. Geophys. Res., doi: 10.1029/2001JA000178, 2002.

Génot, V., Schwartz, S. J., Mazelle C., Balikhin M., and Bauer, T. M.: Kinetic study of mirror mode, J. Geophys. Res., 106, 21 611-21 622, 2001.

Gleaves, D. G. and Southwood, D. J.: Magnetohydrodynamic fluctuations in the earth's magnetosheath at 15:00 LT-ISEE 1 and ISEE 2, J. Geophys. Res., 96, 129-142, 1991.

Hasegawa, A.: Plasma instabilities and non linear effects, Springer Verlag, 1975.

Hellinger, P.,Trávnicek, P., Mangeney, A., and Grappin, R.: Hybrid simulations of the magnetosheath compression: Marginal stability path, Geophys. Res. Lett., 30, 1959-1963, 2003.

Hubert D., Perche, C., Harvey, C. C., Lacombe, C., and Russell, C. T.: Observations of the mirror waves downstream of the quasiperpendicular shock, Geophys. Res. Lett., 16, 159-162, 1989.

Omidi, N. and Winske, D.: Structure of the magnetopause inferred from one-dimensional hybrid simulations, J. Geophys. Res., 100, 11 935-11 955, 1995. 
Pinçon, J. L. and Lefeuvre, F.: Local characterization of homogeneous turbulence in a space plasma from simultaneous measurements of field components at several points in space, J. Geophys. Res., 96, 1789-1802, 1991.

Pinçon, J.-L. and Motschmann, U.: Multispacecraft filtering: general framework, in: Analysis methods for multi-spacecraft data, 65-78, Int. Space Sci. Inst., Bern, Switzerland, 1998.

Rème, H., Bosqued, J. M., and Sauvaud, J. A., et al.: The cluster ion spectrometry (CIS) Experiment, Sp. Sci. Rev., 79, 303-350, 1997.
Rönnmark, K.: Kiruna geophysical institute report, 179, 1982.

Sahraoui, F., Sahraoui, F., Pinçon, J. L., Belmont, G., et al.: ULF wave identification in the magnetosheath: The k-filtering technique applied to cluster II data, J. Geophys. Res., 108(A9), 1335, 2003.

Song, P., Russell, C. T., and Gary, S. P.: Identification of lowfrequency fluctuations in the terrestrial magnetosheath, J. Geophys. Res., 99, 6011-6025, 1994. 